\title{
Energy and Load based Emergency Data Collection Scheme using Mobile Sink for Wireless Sensor Networks
}

\author{
Shivkumar S. Jawaligi \\ Electronics \& Communication Engineering \\ PDA College of Engineering \\ Kalaburagi, India
}

\author{
G. S. Biradar \\ Electonics \& Communication Engineering \\ PDA College of Engineering \\ Kalaburagi,India
}

\begin{abstract}
The extensive Wireless Sensor Networks (WSN), used for environmental monitoring, human body observation, military surveillance, tracking objects and applications. Multi-hop transmission can lead to packet losses, delay, and energy waste due to a large number of re-transmissions and packet (P) drops. The weakness of using a immobile sinks are well known. Advantage of sink mobility for data gathering has drawn a big attention in recent years. Various routing protocols had been developed for achieving network load management in data collecting wireless sensor networks. Mobile sink (MS) reduces the network load and data loss compared to a immobile sink. The main contribution of this paper is to provide a simulation-based study of network load based emergency data collection from harsh environment using mobile sinks. Aim to reduce data loss, minimize energy consumption while establishing path through MS, control emergency mobile sink arrival message storm by zone based WSN. Emergency nodes be capable of minimizing queue overflow through sensor self aggregation and load balancing with neighbors. The focus is on three important configuration specifications: Mobile sink-based data collection, emergencymobile sink arrival message supervision, and sensor self aggregation.
\end{abstract}

\section{General Terms}

Wireless Sensor Networks, Routing protocols.

\section{Keywords}

Mobile Sink; Data Collection; Network Load; Emergency Mobile sink arrival message; Sensor self aggregation;

\section{INTRODUCTION}

Wireless sensor is a transducer whose objective is to sense environmental changes. It detects events in quantities and transmits to the sink node [20]. Measuring such a event concede some effects about objects located and events occurrence in the proximity of the sensor. Disposable sensors can be networked in several applications that require unattended work, e.g., Temperature Sensor, Pressure sensor, Ultrasonic sensor, Humidity Sensor, Gas Sensor, PIR Motion Sensor, the acceleration sensor. Displacement sensors deployed in an area to monitor magnetic fields, gravity, humidity, moisture, vibration, pressure, sound also human body temperature level, glucose level, oxygen level, blood pressure level, heartbeat and so on . As sensor nodes have limited battery life and buffer size [23], energy saving and network load manipulation is utmost important in the design of sensor protocols when harsh environment occurs.

Data collection is the basic function in wireless sensor networks. Sensors may become detached from the network or naturally be destroyed [22], so the network paths have to be shift or re-discovered to readjust for the network to continue its operation [13]. Persistent data collection problem at the time of thoughtless environment through MS considered in this paper. In a static sensor network, an optimal data collection path is generally built to collect the network-wide data. The data collection paths are fixed until it alive and efficiently deliver data to the static sink through multihop. Just before deliver network-wide data to base station (BS) the transmission path need to be build-up or updated from time to time according to the network dynamics [18]. Building the data path often introduces a large volume of communication overheads [17]. Besides, the routing transitions between different data collection paths contain a non-negligible time delay and may lead to discontinuity or even loss of the data packets, which significantly decreases the quality of service (QoS) [16]. Especially in insensitive environment energy and data loss become very high. Traditional multihop based protocols not focus on fundamentally optimizing the routing transitions, with reduced transition overhead, continuous data delivery, minimize data loss and saving energy to increase network life time especially in emergency situation. In effect data transmission by way of multihop routing or tree based routing problems be able to minimized using MS movement in network.

In WSN sensors are not able to recharged or replaced when their batteries drained out of power [14]. The battery weak and drained nodes could cause several problems such as network disconnection, packet loss may possibly leads increasing overheads in network. This increases high energy consumption among network. Thus several WSN studies have engaged in designing efficient methods to conserve the battery power of sensor nodes to extend its lifetime. We focus sensor lifetime extension to deal with the heavy data if occurs in surroundings without packet loss [19]. In general, sensory environment being harsh in some cases. Mobile sinks have been suggested for improving the performance of data collection and handover to the base station in short period [21]. This paper presented a routing scheme that exploits the mobility pattern of the mobile sinks to collect the data from sensors located in zones. we propose LEDMMM Load Based Emergency Data Multiple MS Movement protocol facilitate to form self adaptive network to manipulate the network load.

LEDMMM represents exclusive logical coordinates used to discover mobile sinks without support of GPS devices, which is widely applicable to dynamic sensing network environment. This protocol presents a novel low-complexity dynamic sensor routing to transfer data in harsh environment, which effectively reduces data loss, minimize total energy consumption and increasing network life time. These proposed methods solve the issues of neighborhood node selection for multihop communication. It provides solution for network disconnection, eliminate packet losses, minimize energy consumption, control buffer overflow. 


\section{RELATED WORKS}

A number of approaches exploiting sink mobility for data collection in WSNs have been proposed in recent years Use of MSs for efficient data collection from "sensor islands" spread throughout urban environments [4] discussed and using MobiCluster aims at minimizing the overall network overhead and energy expenditure associated with the data retrieval process and prolonged network lifetime. Routing is called a greedily routable component (GRC) [5] view it as a continuous polygonal area enclosed by a set of boundaries. The area consists of the interior and boundaries of the sensor field, and being continuous means that any two points in the area can be connected by a continuous path within the area. We then consider how to decompose such a polygonal area into a minimum set of greedily routable discussed here. Solve the problem of ubiquitous data collection process for mobile phone users in a wireless sensor network by locally modifying the previously constructed data collection tree with the movement of mobile users in the sensor network [9] to increase the efficiency and scalability in WSN. Endeavors to design a data gathering algorithm using path constrained mobile sink referred to as connectivity-based data collection (CBDC) algorithm[12] . With this algorithm, the major goal is to maximize the network life time whilst satisfying the time and energy constraints of the mobile sink problem as a network utility maximization problem by incorporating multirate wireless communication mechanism between the sensors and the mobile sink a centralized algorithm assuming that the global knowledge of the entire network is available[6]. Also develop a distributed algorithm for the problem without the global knowledge Adaptive sink mobility scheme for data collection and adjusting the local network conditions which minimizes the energy consumption discussed in [11].Network lifetime is defined as the duration from the time sensors start sending data to the data sink to the time when a certain percentage of sensors either run out of battery or cannot send data to the data sink due to the failure of relaying nodes[8]. In the following, for convenience, use M-collector to denote the mobile data collector Distributed clustering technique with multiple links for the wireless sensor networks. The average distance between the sensor nodes and the sink is calculated and the nodes send their location information to the neighboring sinks[2]. The optimal sink places can be found using global information based method. Efficient data collection methods including how to construct data collection structure and how to schedule data collection to achieve near-optimal capacity [3]. This problem was modeled as a sensor data transmission problem, and the design of a feasible data collection route for the mobile sink was analyzed to minimize the energy consumption requirements for a practical system

\section{LEDMMM PROTOCOL DESIGN}

We deal with a large scale, evenly dispersed sensor network deployed in an unrestricted area. We consider the whole sensor network is connected besides the nodes communicate with each other via radio links. Since energy supply of mobile sinks can be reinstate or recharged easily, they are assumed to have sufficient power and it has immense storage capability moreover consistently circulate into the sensing topography. These mobile sinks just as robotics or wireless computing device installed in moving vehicle, have transreceiver to communicate with sensors. To provide efficient and decisive transmissions, network area and node location knowledge will be incorporate with the design and used to guide the virtual zone construction. It does not depend on the shape of the network region, and it is very simple to locate and maintain a zone.

\subsection{Virtual Zone formation}

Virtual zone: Being efficient and robust network maintenance network topography is divided into 4 square zones $(Z)$ from the center $(C)$ point of topology area .

Find center point of entire topology as

$$
\begin{aligned}
& C_{-} x=\frac{\max x}{2} x \\
& C_{-} y=\frac{\max y}{2}
\end{aligned}
$$

Find four zones from the center point

$$
\begin{aligned}
& \text { If }\left(x \leq C_{-} x \& \& y \leq C_{-} y\right) \\
& Z=1 \text {. } \\
& \text { If }\left(x \leq C_{-} x \& \& y>C_{-} y\right) \\
& Z=2 \text {. } \\
& \text { If }\left(x>C \_x \& \& \quad y \leq C \_y\right) \\
& Z=3 \text {. } \\
& \text { If }\left(x>C_{-} x \& \& y>C_{-} y\right) \\
& Z=4 \text {. }
\end{aligned}
$$

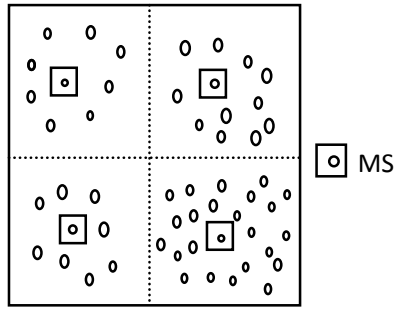

Fig.1: Illustrates MS reached at Stop-Point

Virtual zone ID: A sensor can get its zone ID from its position coordinates $(x, y)$ is the identification of zone by each sensor.

\section{Algorithm 1. MS Moving strategies}

/*_-Initialization___*/

1. msg.seqN $\leftarrow 0$;

2. msg.hopC $\leftarrow 0$;

3. Initial Energy $\leftarrow$ 100joules

4. Initial buffer size $\leftarrow 50$

/*__Moving strategies___*/

5. Broadcast MS_beacon

6. Receive Stop-Point with Mobile_Sink_Arrival_Request

7. Check state: 1. Normal_Mode 2. Emergency_Mode

8. Normal_Mode : Exchange moving zone info to other MS

9. Collect data upto LoR and move to next zone

10. Move each zone in regular interval 


\section{1. while MS Not get enough data or timeout do}

\section{Move to next Stop-Point}

13. Stop upto LoR duration broadcast Stop-Point message

14. $m s g . s e q N \leftarrow m s g . s e q N+1$;

15. If State :Emergency_Mode skip regular moving zone

16. Move Emergency Mode enabled node zone direction

17. Concurrently listen for data $P$;

18. end while

\section{End data gathering process and exit;}

In the LEDMMM algorithm, vectors called Stop References. It represent logical coordinates in a network. The stop reference maintained by each node is used as a location indicator for packet forwarding

\subsection{Neighbor Maintenance}

Neighbor connectivity retains when there is a change in topology through the local broadcast hello message periodically which carries node attributes. Each node build neighbor table which contains the following information about its neighbor nodes $(\mathrm{NN})$.

NN_ ID | Current queue length

Since sensor node has queue limit, upto the q_thresh it can store the sensed data packets subsequently buffer overflow can arise in case of intensive traffic load. Therefore each sensor knows its q_limit and exchange through hello message and update in neighbor table. Assuming that sensor has reached maximum q_thresh limit it switch to active mode $A M$ and enclose Emergency_Mode in next hello message.

\subsection{Mobile Sink Movement}

At beginning, $M S$ moves into the zones around network area by low speed, and broadcast $M S$ _beacon message to announce its presence in addition keeps hearing for Mobile_Sink_Arrival_Request to know the zone ID and StopPoint. MS moves to the zone based on first received query and reside at Stop-Point upto LoR duration. Broadcast Data_Report_Requisition message with "Stop-Point" to the zone nodes, subsequently finished data collection thereupon MS moves to next zone in Normal Mode. Data collection time $D t$ from a zone given as

$$
D t=\frac{d i s t}{S}
$$

Data transferring delay time from sensor to $M S$ taken as dist. Since network divided as zones every sensor get a chance to transfer data directly to $M S$ with one hop communication. S reflects rate of data transmission. each zone prefer least one Stop-Point for MS. Suppose the network is dense, pick more than one Stop-Point upon equal distance. Compute length of rotation - LoR for $M S$ at each zone based on $M S$ speed (SD) and stop time to collect data $D t$.

$$
L o R=S D * D t
$$

As if two adjacent Stop-Point distances between any two consecutive Stop-Points are same otherwise, the hop count information will not be significantly different. Soon after compute $M S$ movement direction randomly to next zone in Normal_Mode. Each sensor aware of its zone ID and StopPoint location of its zone. Send Stop-Point message via Mobile_Sink_Arrival_Request to mobile sink all the way through network center-point near sensor. Mobile sink receives and stores the Stop-Point and its next movement direction in between zones in Normal_Mode. The MS can observe its speed and direction of movement.

\subsection{Mobile Sink Arrival}

After reaching at the Stop-Point, the MS will broadcast Data Report_Requisition message to sensors, which will in turn to send the sensed data to the mobile sink.

\subsection{Emergency Mode EM and Sensor Self Aggregation}

Harsh environment emerged in any part of the zone or entire zone, because of the atmospheric conditions, continual data diversity sensed by the sensors will be incredible. Being wireless nature each node get minimum chance to transmit the sensed packets in view of channel scarcity. If sensor does not get channel to transmit data or if $M S$ not arrived that zone at this critical time each node packet count will be increased. Though packets count raised in queue so that waiting time starts until it transmits in queue. Where packets are dropped because of queue overflow which is universal problem. Exclusively in sensor network,s packet loss results increased overhead in terms of energy wasted to re-transmit a packet which was dropped. The proposed view presents sensor self aggregation to manage the queue waiting time personally and reduce packet loss. Each node watch its queue limit and when its crossed the q_thresh_starts self aggregation to balance the heavy load in sensing region and store the Mzx_Sense_Value from the stored data within a period of time and drop same kind of data from queue.

This self aggregation reduces the severity of losses and gives ability to manage the queue to receive new arrival packets. This improves the intensity and effectiveness of current queue.

$$
\begin{aligned}
& \text { if (current_q_size increased) } \\
& \text { if current_q_size > q_thresh } \\
& \text { start Emergency_Mode in hello packet }
\end{aligned}
$$

based on hello message transmission its immediate neighbors knows its previous neighbor suffered because of heavy loadin queue. To protect the node from packet loss neighboring nodes starts Emegency_Mobile_Sink_Arrival_Request message.

\section{MULTIPLE MOBILE SINK MOVEMENT}

Multiple mobile sink used to collecting data in dense network within short time [15]. MS ID, Stop-Point Msg_ID, is added to each Stop-Point message to identify them from different MS. Mobile Sink moves each Stop-Point in Normal_Mode wait upto LoR period as well Broadcast data_ Report_Requisition Stop-Point message along with its StopPoint. Sensors received Stop-Point message and Increment MS count if it receives more than one Stop-Point message. Instead of using only one Stop-Point, a sensor node maintains multiple Stop-Point messages that each corresponds to a different mobile sink. Supposing that $M S$ receives Emegency_Mobile_Sink_Arrival_Request it changes the current Stop-Point movement to Emergency_Mode enabled node zone location to collect data. If more than one $M S$ receives Emegency_Mobile_Sink_Arrival_Request message each $M S$ exchange its start time and moving zone id to another $M S$ to avoid multiple $M S$ visit in emergency area through its high transmission range. Data holding time by 
$M S$ computed through $L o R+$ traveling all nodes in zone to collect data and coming back to the base station. Through this each mobile sink reduce its Job completion time (JCT) and return to BS shortly Through this assignment $\mathrm{n}$ all Mobile sinks has equal option to balance the network data collection even in most severe situations.

Mobile sink Stop-Point count ( SC)

MS movement count ( MMC)

Distance between each nodes -( dij)

Speed of MS ( $S D)$

$$
\begin{array}{r}
M S_{-} j o b \_h a n d l i n g \_C o u n t \quad-(j h c) \\
J C T=\frac{J h c * S C \sum(M M C * d i j)}{S D}
\end{array}
$$

Mobile sink moving distance computed as

$$
M S M D=\sum \forall \operatorname{nodes}\left(M M_{C} * d i j\right)
$$

\subsection{Stop-Point Reference}

Creates Logical co-ordinates of network [15]. At beginning all sensor nodes Stop-Point references are initialized to [ -1 , $-1, \ldots .,-1]$. A special variable $\lambda$ that is used to track the latest message sequence number is also set to -1 . After the mobile sink $\mathrm{S}$ enters the field, it randomly select a place as its first Stop-Point and broadcasts a Stop-Point_Message to all the sensor nodes . The Stop-Point_Message (SeqN, Stop-Point ), is set to $(1,1)$, indicating that this is the first StopPoint_Message 1, and the hop count (hopC) to $M S$ is 1 . The nodes nearest to $M S$ will be the first one to hear this message. By comparing with $\lambda$, if this is a new message, then $\lambda$ will be updated by the new sequence number. Each node maintain Stop-Point information from Stop-Point_Message. Update hopC and if network is dense this StopPoint_Message will be rebroadcast with the same sequence number and an incremented hopC. The same procedure repeats at all the other nodes and update their hopC distances to MS Stop-Point. If a node receives a Stop-Point message with a sequence number equals to $\lambda$, but has a smaller hopC than it has already recorded, then the last hopC field in its Stop-Point is updated, and this Stop-Point message is rebroadcast with the same sequence number and an incremented hopC. Stop-Point message that has sequence number less than $\lambda$ will be discarded to eliminate flooding messages in the network.

\section{Algorithm 2. Stop-Point message update}

\section{1: while Data gathering process $i$ do}

2: /*-Receive a Stop-Point message_*/

3: if msg.seq $N>\lambda$ then

4: $\lambda \leftarrow$ msg.seq

5: Update seqN ;

6: $m s g . h o p C \leftarrow m s g . h o p C+1$;

7: Rebroadcast message ;

8: else if msg.seqN $=\lambda$ then;

9: $\quad$ Compare hopC < previous message

10: $\quad$ update msg.hopC ;

\section{1: $\quad$ Rebroadcast message;}

12: else ;

13: $\quad$ Discard the message;

14: $\quad$ end if

15: else if msg.seq $N<\lambda$ then

16: $\quad$ Discard the message;

17: $\quad$ end if

18: $\quad$ end while

After each node in the network received distinct Stop-Point message, the logical coordinate space is established.

\subsection{Mobile Sink Announcement}

$M S$ moves entire network to collect sensed data from sensors It send Announcement and start data_timer, every $2 \mathrm{sec}$ once send collected data from sensor to BS done by $M S$. MS send Data_Report_Requisition message with its location If emergency Message arrived immediately moves to that area and broadcast Emergency_Data_Report_Requisition to gather data from Emergency Node (EN) also nearby nodes. Receiving node drop packet if seqN less than previous one. If seqN is new update Sink_Routing_Table (Sr_table), Previous node (PrevN) and distance (D) also update sequence number table SeqN-Table

\section{ANNOUNCEMENT RECEIVING :}

$$
\begin{aligned}
& \text { If node! = sender } \\
& \text { seqN < Received seqN } \\
& \text { DROP P } \\
& \text { else } \\
& \quad \text { Update Sr_table } \\
& \quad \text { Update Sr_table with hopC, PrevN, D } \\
& \quad \text { Update SeqN-Table } \\
& \text { Compute D between sensor and } M S
\end{aligned}
$$

\subsection{Load Balancing}

If Emergency_Mode enabled node not received any Emergency_Data_Report_Requisition message in a short period of time it will verify neighbours a_thresh from hello message. If any neighborhood node has more free space in queue EN send some packets to that node to avoid packet loss.

$$
\text { If }\left(N N_{-} q \_ \text {thresh > EN_q_thersh }\right)
$$

forward data to neighbors to balance the queue in network.

\subsection{Data Collection}

Sensors generate data with random interval with random rate depends on environment. $M S$ send data collection message with time stamp - Time and location computed with distance(D) to receiving nodes and speed (SD)

$$
\text { Time }=D / S D
$$

receiving nodes stores ID of $M S$ then forward stored packet from buffer to $M S$ then Remove data from queue. $M S$ has more buffer size than any other nodes and re-powered option. So that it collects data and moves to BS to handover 
collected data and free its queue space and update power also.

\section{SIMULATION RESULTS}

A simulation yields many feasible values for the outcomes as per protocol and environmental impact. The task of simulation investigation is to summarize and evaluate the results, in a way that will yield utmost insight and help with decision-making about protocol behaviors.

\section{ENERGY CONSUMTION Vs NETWORK SIZE}

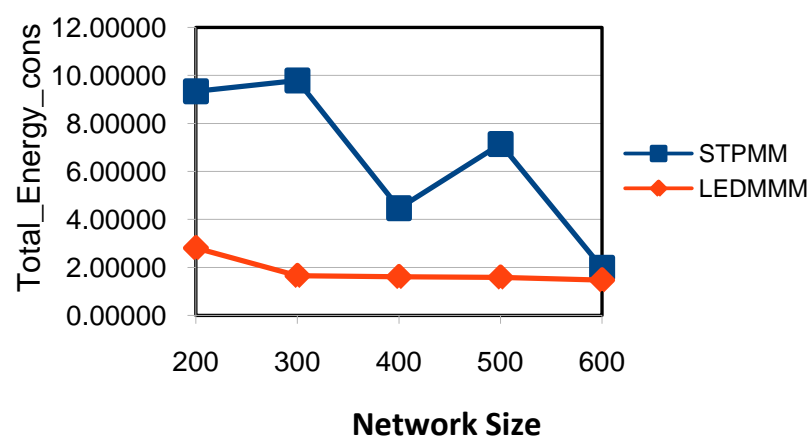

Fig.2.Examines consumed energy between STPMM and LEDMMM

LEDMMM focus on providing the lowest energy consumption. A sensor node should be small in size, consume extremely low energy, operate in high densities in unattended area and adaptive to the environment to save energy.

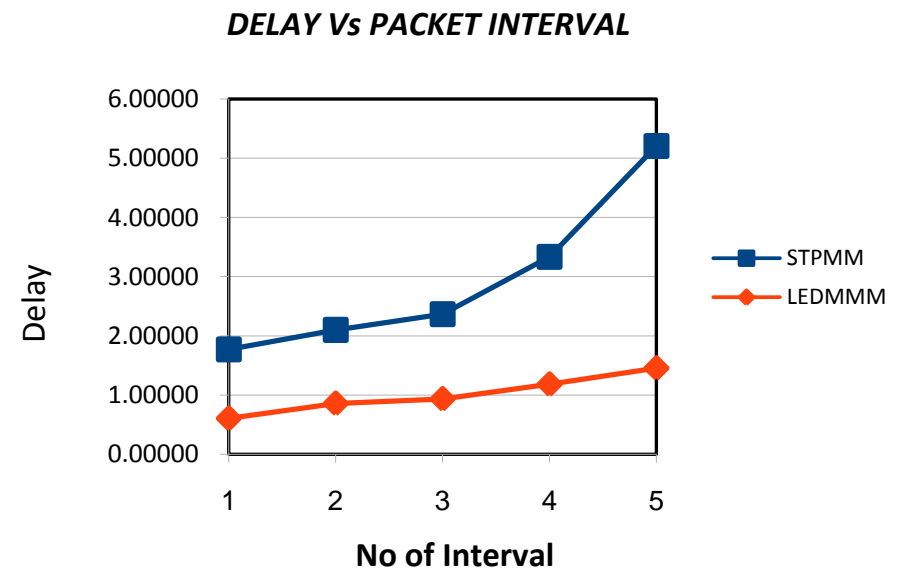

Fig.3 Define delay between source and destination

Network delay is an important design and performance characteristic of a computer network or telecommunications network. The delay of a network specifies how long it takes for a bit of data to travel across the network from one node or endpoint to another. 


\section{DROPPING RATIO VS PACKET INTERVAL}

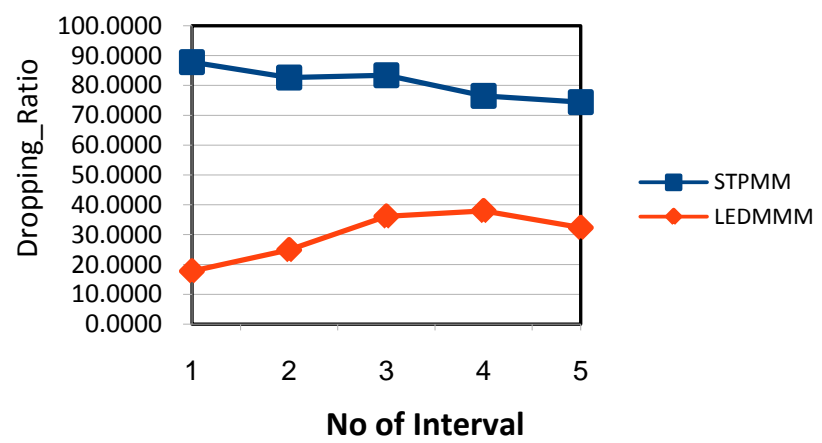

Fig.4. Analyze the drop ratio of STPMM and LEDMMM

Knowing packet drop ratio helps to determine the problem It can be caused by a number of reasons, network disconnection, Queue overflow, Channel scarcity. So using a packet drop ratio it is a part of the troubleshooting process.

\section{CONCLUSION}

The above works mainly focus on reduce energy consumption, packet loss, minimize emergency mobile sink arrival message to manage the network load and minimize energy consumption. It uses logical coordinates to infer distances, and In addition, establishes data reporting routes by greedily shortest path to the destination for each transmission.

\section{REFERENCES}

[1] Luo Mai, Longfei Shangguan, Chao Lang, Junzhao Du, Hui Liu, Zhenjiang Li, and Mo Li, "Load Balanced Rendezvous Data Collection in Wireless Sensor Networks, 2011 Eighth IEEE International Conference on Mobile Ad-Hoc and Sensor Systems,2011

[2] Manisekaran, S.V. and R. Venkatesan, "Distributed Cluster Based Routing Technique with Multiple Sinks for Wireless Sensor Networks," American Journal of Applied Sciences 9 (8): 1242- 1250, 2012 ISSN 1546-9239, 2012.

[3] Siyuan Chen, Minsu Huang, Shaojie Tang, Student Member, IEEE, and Yu Wang, Senior Member, IEEE, "Capacity of Data Collection in Arbitrary Wireless Sensor Networks," IEEE Transactions On Parallel And Distributed Systems, Vol. 23, January 2012.

[4] Charalampos Konstantopoulos, Grammati Pantziou, Damianos Gavalas, Aristides Mpitziopoulos, and Basilis Mamalis, "A Rendezvous-Based Approach Enabling Energy-Efficient Sensory Data Collection with Mobile Sinks," IEEE Transactions On Parallel And Distributed Systems, vol. 23, May 2012.

[5] Guang Tan, Member, ACM and Anne-Marie Kermarrec, "Greedy Geographic Routing in Large-Scale Sensor Networks: A Minimum Network Decomposition Approach,"IEEE/ACM Transactions On Networking, Vol. 20, June 2012.

[6] Xiaojiang Ren, Weifa Liang, " The Use of A Mobile Sink for Quality Data Collection in Energy
Harvesting Sensor Networks," 2013 IEEE Wireless Communications and Networking Conference (WCNC): NETWORKS, 2013.

[7] M. Ma and Y. Yang, "Data Gathering in Wireless Sensor Networks with Mobile Collectors," Proc. IEEE Int'l Symp. Parallel and Distributed Processing (IPDPS), pp. 1-9, Apr. 2008.

[8] A.A. Somasundara, A. Ramamoorthy, and M.B. Srivastava, "Mobile Element Scheduling for Efficient Data Collection in Wireless Sensor Networks with Dynamic Deadlines," Proc. IEEE 25th Int'l Real-Time Systems Symp. (RTSS), pp. 296-305, 2004.

[9] F. Ye, G. Zhong, S. Lu, and L. Zhang, "Gradient Broadcast: A Robust Data Delivery Protocol for Large Scale Sensor Networks," Wireless Networks, vol. 11, no. 3, pp. 285-298, 2005.

[10] Ramarajan C, Ajitha P, "Data Gathering Mechanisms with Multiple Mobile Collectors in Wireless Sensor Network," IOSR Journal of Computer Engineering (IOSR-JCE) e-ISSN: 2278- 0661, pISSN: 2278-8727, Volume. 16,Issue 1, PP 01-05, Feb. 2014.

[11] P. MADHUMATHY, D. SIVAKUMAR, "Mobile Sink Based Reliable and Energy Efficient Data Gathering Technique for WSN," Journal of Theoretical and Applied Information Technology, vol. 61 No.1, 10th March 2014.

[12] Abdullah I. Alhasanat*, Khaled D. Matrouk, Haitham A. Alasha'ary, Ziad A. Al-Qadi, "Connectivity-Based Data Gathering with Path-Constrained Mobile Sink in Wireless Sensor Networks," Wireless Sensor Network, 2014, 6, 118- 128, Published Online June 2014.

[13] S. Basagni, A. Carosi, E. Melachrinoudis, C. Petrioli, and Z.M.Wang, "Controlled Sink Mobility for Prolonging Wireless Sensor Networks Lifetime," ACM/Elsevier Wireless Networks, vol. 14, pp. 831-858, 2007.

[14] C. Chou, K. Ssu, H. Jiau, W. Wang, and C. Wang, "A Dead-End Free Topology Maintenance Protocol for Geographic Forwarding in Wireless Sensor Networks," IEEE Trans. Computers, vol. 60, no. 11, pp. 1610-1621, Nov. 2010. 
[15] Xinxin Liu, , Han Zhao, , Xin Yang, and Xiaolin Li, SinkTrail: A Proactive Data Reporting Protocol for Wireless Sensor Networks IEEE Transactions On Computers, Vol. 62, No. 1, January 2013

[16] K. Fodor and A. Vida'cs, "Efficient Routing to Mobile Sinks in Wireless Sensor Networks," Proc. Third Int'l Conf. Wireless Internet (WICON), pp. 1-7, 2007.

[17] R. Fonseca, S. Ratnasamy, J. Zhao, C.T. Ee, D. Culler, S. Shenker, and I. Stoica, "Beacon Vector Routing: Scalable Point-To-Point Routing in Wireless Sensornets," Proc. Second Conf. Networked Systems Design and Implementation (NSDI), pp. 329-342, 2005.

[18] Q. Huang, C. Lu, and G. Roman, "Spatiotemporal Multicast in Sensor Networks," Proc. First ACM Conf. Embedded Networked Sensor Systems (SenSys), pp. 205-217, 2003.

[19] C. Intanagonwiwat, R. Govindan, and D. Estrin, "Directed Diffusion: A Scalable and Robust Communication Paradigm for Sensor Networks," Proc. MobiCom, pp. 56-67, 2000
[20] M. Keally, G. Zhou, and G. Xing, "Sidewinder: A Predictive Data Forwarding Protocol for Mobile Wireless Sensor Networks," Proc. IEEE Sixth Ann. Comm. Soc. Conf. Sensor, Mesh and Ad Hoc Comm. and Networks (SECON), pp. 1-9, June 2009.

[21] Z. Li, N. Wang, A. Franzen, P. Taher, C. Godsey, H. Zhang, and X. Li, "Practical Deployment of an In-Field Soil Property Wireless Sensor Network," Computer Standards and Interfaces, vol. 33, pp. 13- 23, 2011.

[22] B. Liu, W. Ke, C. Tsai, and M. Tsai, "Constructing a Message-Pruning Tree with Minimum Cost for Tracking Moving Objects in Wireless Sensor Networks is Npcomplete and an Enhanced Data Aggregation Structure," IEEE Trans. Computers, vol. 57, no. 6, pp. 849-863, June 2008.

[23] T. Park, D. Kim, S. Jang, S. eun Yoo, and Y. Lee,"Energy Efficient and Seamless Data Collection with Mobile Sinks in Massive Sensor Networks," Proc. IEEE Int'l Symp. Parallel and Distributed Processing (IPDPS),pp.1-8,May-2009. 